\title{
RIGIDITY AND DYNAMICS AROUND MANIFOLDS OF NEGATIVE CURVATURE
}

\author{
Chengbo Yue
}

This survey grew out of a series of talks at A. Katok's seminar on "geometric rigidity and smooth group actions". A complete survey on manifolds of negative curvature should include 1) Kanai [K], Feres-Katok [FK1], $[\mathrm{FK} 2],[\mathrm{F}]$ and Benoist-Foulon-Labourie's [BFL] [BF] work on the regularity of Anosov foliations; 2) The results of Hamenstädt [H4] [H5] on entropy rigidity (as a generalization of Mostow rigidity); 3) The work of Croke [C] and Otal $[\mathrm{O}]$ on marked length spectrum; 4) Besson, Courtois and Gallot's work $[\mathrm{BCG}]$ on minimal entropy of locally symmetric spaces, and many others. However, the lack of time and space forces ourselves to restrict to problems of more analytical nature. Most of the problems discussed here seem to have originated from [K1]: various measures associated with the geodesic flow, entropy like invariants of the Riemannian metric. They turn out to be closely related to many other rigidity problems: regularity of the Anosov foliation, marked length spectrum, etc. In as much as this is a survey, it should be obvious that not all the proofs are given. However, we do not hesitate to provide a detailed explanation of the main ideas whenever possible. I also take this opportunity to state some new results and write down some open problems. We hope the bibliography to be a helpful guide into the evolution of the subject.

\section{Basic constructions}

\subsection{The meaning of non-positive curvature.}

Consider a surface of negative curvature and let $\Delta$ be a geodesic triangle with angles $\alpha_{1}, \alpha_{2}, \alpha_{3}$. By the Gauss-Bonnet theorem, $\alpha_{1}+\alpha_{2}+\alpha_{3}=\pi+$ $\int_{\Delta} K<\pi$ where $K$ is the sectional curvature. This means that in negative curvature the two geodesics $\gamma_{1}, \gamma_{2}$ diverge faster than in the Euclidean situation. In higher dimensions, we can think of Jacobi fields which sweep infinitesimal triangles. Let $J(t)$ be a Jacobi field along a geodesic $v(t)$. So it satisfies the Jacobi equation $J^{\prime \prime}=-R(J, \dot{v}) \dot{v}$ where $R$ is the curvature

Received January 14, 1994. 
operator. Then $\left\langle J, J^{\prime}\right\rangle^{\prime}=\left\langle J, J^{\prime \prime}\right\rangle+\left\langle J^{\prime}, J^{\prime}\right\rangle=-\langle R(J, \dot{v}) \dot{v}, J\rangle+\left\|J^{\prime}\right\|^{2} \geq\left\|J^{\prime}\right\|^{2}$ by non-positive curvature. Thus, using the Schwarz inequality,

$$
\|J\|^{\prime \prime}=\left(\frac{\left\langle J, J^{\prime}\right\rangle}{\|J\|}\right)^{\prime}=\frac{\left\langle J, J^{\prime}\right\rangle^{\prime}\|J\|^{2}-\left\langle J, J^{\prime}\right\rangle^{2}}{\|J\|^{3}} \geq \frac{\left\|J^{\prime}\right\|^{2}\|J\|^{2}-\left\langle J, J^{\prime}\right\rangle^{2}}{\|J\|^{3}} \geq 0,
$$

and we get the following result (see $[\mathrm{Kl}]$ for example).

Proposition 1.1. $i)\|J\|$ is a convex function. ii) If $v_{1}(t), v_{2}(t)$ are two geodesics, then $d\left(v_{1}(t), v_{2}(t)\right)$ is a convex function of $t$.

1.2. Some comments on manifolds of non-positive curvature. Let $G$ be a semi-simple group of non-compact type. Let $H \subset G$ be a maximal compact subgroup. Then $\widetilde{M}=G / H$ admits a $G$-invariant metric $g$. By the theorem of E. Cartan, $K(g) \leq 0$ and $K$ is strictly negative if and only if $\operatorname{rank}_{\mathbb{R}} G=1$. In fact, one can define the rank of any manifold of nonpositive curvature by $\operatorname{rank}(M) \triangleq \min _{v \in S M}\{$ dimension of the space of Jacobi fields parallel along the geodesic $v(t)$, where $v(t)$ is the geodesic with initial velocity $v: \dot{v}(0)=v\}$.

Theorem 1.2. Let $M$ be complete, $\operatorname{vol}(M)<\infty,-a^{2} \leq K \leq 0$ for some positive number a. Assume that the universal cover $\widetilde{M}$ is irreducible (i.e. $\widetilde{M}$ does not split into a Riemannian product). Then (Ballmann, Brin, Burns, Eberlein, Spatizer [BBE] [BBS] [BA] [BS])

i) Either $\widetilde{M}=G / H$, as described above (symmetric).

ii) or $\operatorname{rank}(M)=1$.

Remark. By theorem 1.2 and Margulis' arithmeticity theorem all finite volume manifolds with rank $\geq 2$ and nonpositive curvature arise in a simple way-they are the quotients $\Gamma \backslash G / H$ where $\Gamma$ is a discrete subgroup produced usually by arithmetic constructions. On the contrary, there are many rank-1 manifolds of nonpositive curvature which are not the quotients of Lie groups. For example, there are closed manifolds with arbitrarily pinched negative curvature $-1 \leq K \leq-1+\epsilon$ which are not homeomorphic to any manifold of constant negative curvature (see $[\mathrm{GT}]$ ). However, the geodesic flow of a rank-1 manifold is almost Anosov. The geometric characteristics of the metric are reflected in the asymptotic properties of this flow.

1.3. Notations. From now on we consider a closed $n$-dimensional Riemannian manifold $M$, with negative sectional curvature $-\infty<-b^{2} \leq K \leq$ $-a^{2}<0$. We denote by

$$
\widetilde{M} \text { the universal cover of } M \text {; }
$$


$S M($ resp. $S \widetilde{M})$ the unit tangent bundle of $M($ resp. $\widetilde{M})$;

$\Gamma$ the fundamental group $\Gamma=\pi_{1}(M)$;

$v(t)$ the unit speed geodesic on $M$ or $\widetilde{M}$ with initial velocity $\dot{v}(0)=v$.

The geodesic flow $g^{t}$ is defined on $S M$, by $g^{t}(v)=\dot{v}(t)$. The Jacobi equation $\ddot{J}+R(J, v) v=0$ is exactly the linear approximation of the geodesic flow (see $[\mathrm{Kl}]$ ). Thus, by the discussion in 1.1, the norm $\|J(t)\|$ of each Jacobi field $J(t)$ is a strictly convex function. Let us denote by $E^{s}$ (resp. $E^{u}$ ) the space of all Jacobi fields $J(t)$ which satisfy $\|J(t)\| \rightarrow 0$ as $t \rightarrow \infty$ (resp. $t \rightarrow-\infty$ ). Then by the usual identification $([\mathrm{Kl}])$ of the tangent space of $S M$ with the space of Jacobi fields, we obtain a continuous decomposition $T S M=E^{s} \oplus E^{u} \oplus \mathbb{R} X$ where $X$ is the geodesic spray, such that for some constant $A>0$, if $\xi \in E^{s}$, then $A^{-1} e^{-b t} \leq\left|d g^{t}(\xi)\right| \leq$ $A e^{-a t}|\xi|$ for all $t \in \mathbb{R}$ and if $\xi \in E^{u}$, then $A^{-1} e^{a t}|\xi| \leq\left|d g^{t}(\xi)\right| \leq A e^{b t}|\xi|$ for all $t \in \mathbb{R}$. In other words, $g^{t}$ is an Anosov flow. Under this splitting, the differential of the geodesic flow along the $E^{s}$-direction $\left.d g^{t}\right|_{E^{s}}$ is represented by a fundamental matrix $Y(t)$ which satisfies the Jacobi equation $\dot{Y}+R(Y, v) v=0$. We can set $U(t)=\dot{Y}(t) Y^{-1}(t)$. Then $U$ is exactly the second fundamental form of the horosphere which is positively asymptotic to $v$ (see $[\mathrm{Kl}]$ ) and it satisfies the Ricatti equation $-\dot{U}+U^{2}+R=0$.

1.4. The ideal sphere $\partial \widetilde{M}$ and its regularity. Since $g^{t}$ is Anosov, the distance between two geodesics in $\widetilde{M}$ either tends to 0 or tends to infinity as $t \rightarrow \infty$. In the former case they are called asymptotic. The ideal boundary $\partial \widetilde{M}$ is defined as the set of equivalence classes of asymptotic geodesics of $\widetilde{M}$. Given a point $x \in \widetilde{M}$, then for any $v \in S_{x} M$, there is a unique equivalence class corresponding to $v(t)$, which we denote by $v(\infty)$, thus we have a bijection

$$
S_{x} \widetilde{M} \stackrel{P_{x}}{\longrightarrow} \partial \widetilde{M}
$$

We can check that the topology on $\partial \widetilde{M}$ that makes $P_{x}$ a homeomorphism is independent of the point $x$. This is called the sphere topology.

Proposition 1.4.1 ([A] $[\mathrm{AS}])$. $\partial \widetilde{M}$ has a $C^{\alpha}$ (i.e. $\alpha$-Hölder)-structure, where $\alpha=\frac{a}{b}$.

Proof. Let $o, x_{1}, x_{2}$ be three points in $\widetilde{M}$ and $d$ be the Riemannian distance. By the Toponogov theorem [CE]: if $d\left(o, x_{1}\right)=d\left(o, x_{2}\right)=r$, then $2 r+$ $\frac{2}{a}(\ln \theta-1) \leq d\left(x_{1}, x_{2}\right) \leq 2 r+\frac{2}{b}(\ln \theta+1)$ for all $r$ large enough and $\theta$ small enough, where $\theta$ is the angle spanned by the geodesics $\overrightarrow{o x_{1}}$ and $\overrightarrow{o x_{1}}$.

Now let $v, w \in S_{x_{1}} \widetilde{M}, \theta=\angle(v, w)$ and $\theta_{t}=\angle\left(x_{2} ; v(t), w(t)\right)$ (i.e. the angle at $x_{2}$ spanned by the two points $v(t)$ and $\left.w(t)\right)$. Then again by 
the Toponogov theorem, if $t$ is large enough, $\frac{2}{a}\left(\ln \theta_{t}-1\right)+2 t+C_{1} \leq$ $d(v(t), w(t)) \leq 2 t+\frac{2}{b}(\ln \theta+1)$ for some constant $C_{1}=C_{1}\left(x_{1}, x_{2}\right)$. Thus $\theta_{t} \leq C_{2} \theta^{\frac{a}{b}}$ for some constant $C_{2}$.

\section{Remarks.}

1. It is easy to check that the $C^{\alpha}$-regularity of $\partial \widetilde{M}$ is equivalent to the $C^{\alpha}$-regularity of the Anosov splitting $T S M=E^{s} \oplus E^{u} \oplus \mathbb{R} X$.

2. If $\operatorname{dim} M=2$, or if $\frac{a}{b}>\frac{1}{2}$ (i.e. $\frac{1}{4}$-pinching) then $\partial \widetilde{M}$ carries a $C^{1}$ structure $([\mathrm{HP}])$. See also $[\mathrm{Ha} 1]$ for further results. However, our argument here only gives a $C^{\frac{1}{2}}$-structure.

3. For more delicate regularity properties in dimension 2 , see $[\mathrm{HK}]$.

4. In [BFL], Benoist, Foulon and Labourie, generalizing works of Kanai, Feres and Katok, prove that for a closed Riemannian manifold of negative curvature, if the ideal boundary has a $C^{\infty}$ structure then its geodesic flow is time preserving $C^{\infty}$ conjugate to the geodesic flow of a locally symmetric space of negative curvature. Furthermore, the $C^{\infty}$-condition was reduced to some $C^{k}, k<\infty$, in [Ha2].

1.4.2. Busemann functions and horospheres. For each $v \in S \widetilde{M}$, there corresponds a Busemann function $\rho_{v}(y) \stackrel{\text { def }}{=} \lim _{t \rightarrow \infty} d(v(t), y)-t$. Sometimes one also writes it as $\rho_{x, \xi}(y)$, where $\xi=v(\infty)$ is the asymptotic class of the geodesic $v(t)$. This is a smooth function of $y$. The level set of the Busemann function at $v$ through $y$ is called the horosphere centered at $\xi$ passing through $y$. We denote it by $H(y, \xi)$ or $H(v)$ if $v \in S_{y} M$ and $v(\infty)=\xi$.

1.4.3. Action of $\Gamma$ at infinity. Every isometry $\gamma \in \Gamma$ extends to a homeomorphism of $\partial \widetilde{M}$ naturally: $\gamma(v(\infty)) \stackrel{\text { def }}{=} \gamma(v)(\infty), v \in S M$. If $\Gamma$ is cocompact, then it is well known $[\mathrm{E}]$ that the $\Gamma$-action on $\partial \widetilde{M}$ is minimal, i.e., every $\Gamma$-orbit is dense in $\partial \widetilde{M}$. $\Gamma$ also acts naturally on $\partial \widetilde{M} \times \partial \widetilde{M}$ by $\gamma(\xi, \eta)=(\gamma \xi, \gamma \eta)$. Observe that the space of geodesics on $\widetilde{M}$ is canonically identified with $\partial \widetilde{M} \times \partial \widetilde{M} \backslash$ diagonal. There is also a natural 1-1 correspondence between the following two sets: $\left\{g^{t}\right.$-invariant measures on $\left.S M\right\} \longleftrightarrow$ $\{\Gamma$-invariant measures on $\partial \widetilde{M} \times \partial \widetilde{M} \backslash$ diagonal $\}$.

1.5. Patterson-Sullivan measure at infinity. Next we shall construct two families of measures on $\partial \widetilde{M}$. (i) the Patterson-Sullivan measures, using the lattice $\Gamma$-action; and (ii) the harmonic measures using potential theory. Observe that all these constructions are apparently unrelated with the geodesic flow. 
Fix two points $x, y \in \widetilde{M}$ and consider the Poincaré series $g_{s}(x, y)=$ $\sum_{\gamma \in \Gamma} e^{-s d(x, \gamma y)}$. Define the critical exponent $\delta(\Gamma) \stackrel{\text { def }}{=} \delta \stackrel{\text { def }}{=} \sup \left\{s>0 \mid g_{s}(x, y)\right.$ diverges at $s\}$. Now let $S_{k}$ be the number of orbit points $\Gamma y$ in the annulus $B\left(x, k+\frac{1}{2}\right) \backslash B\left(x, k-\frac{1}{2}\right)$ where $B(x, r)$ denotes the geodesic ball centered at $x$ of radius $r$, then $g_{s}(x, y) \sim \sum_{k=0}^{\infty} S_{k} e^{-k s}$. Therefore

$$
\begin{aligned}
\delta=\varlimsup_{k \rightarrow \infty} \frac{\log S_{k}}{k} & =\varlimsup_{k \rightarrow \infty} \frac{\log \operatorname{vol}\left[B\left(x, k+\frac{1}{2}\right) \backslash B\left(x, k-\frac{1}{2}\right)\right]}{k} \\
& =\varlimsup_{k \rightarrow \infty} \frac{\log \operatorname{vol}(B(x, k))}{k} .
\end{aligned}
$$

Now we fix a reference point $x_{0}$ and consider the family of measures on $\widetilde{M}$ :

$$
\mu_{x}^{s}=\frac{1}{g_{s}\left(x_{0}, x_{0}\right)} \sum_{\gamma \in \Gamma} e^{-s d\left(x_{0}, \gamma x\right)} \delta_{\gamma x},
$$

where $\delta_{\gamma x}$ is the Dirac measure at $\gamma x$. Using the triangle inequality it is easy to see that $e^{-s d\left(x_{0}, x\right)} g_{s}\left(x_{0}, x_{0}\right)<g_{s}\left(x_{0}, x\right)<e^{+s d\left(x_{0}, x\right)} g_{s}\left(x_{0}, x_{0}\right)$. Therefore $\left\{\mu_{x}^{s}\right\}_{s>\delta}$ is a family of finite measures on $\widetilde{M}$ with uniformly bounded total mass. Let $\mu_{x}=\lim _{s_{j} \rightarrow \delta+} \mu_{x}^{s_{j}}$ be a weak limit. It is easy to see that $\mu_{x}$ is concentrated on $\partial \widetilde{M}$ and that for any other point $y \in \partial \widetilde{M}$, $\mu_{y}=\lim _{s_{j} \rightarrow \delta+} \mu_{y}^{s_{j}}$ also exists. Moreover we have for $\xi \in \partial \widetilde{M}$,

$$
\frac{d \mu_{y}}{d \mu_{x}}(\xi)=e^{-\delta \rho_{x, \xi}(y)}
$$

where $\rho_{x, \xi}(y)$ is the Busemann function (see 1.4.2).

1.6. Dirichlet Problem and Harmonic Measures. (See the references $[\mathrm{A}],[\mathrm{S} 1],[\mathrm{AS}])$. One example of how negative curvature affects geometry is given by the behavior of the Laplacian. Let $r(y)=d(x, y)$ be the distance function, then we have

$$
\Delta e^{-\delta r}=\left[\delta^{2}-\delta \cdot \frac{\theta^{\prime}}{\theta}\right] e^{-\delta r}
$$

here $\theta(x, y)$ is given by $d^{*}\left(\exp _{x}(\cdot)\right) d m(y)=\theta(x, y) \cdot d \sigma$, where $d^{*}$ denotes the Jacobian, $d m(y)$ is the Riemannian volume on $\widetilde{M}$ and $d \sigma$ is the Euclidean volume on $T_{x} \widetilde{M}$ and $\frac{\theta^{\prime}}{\theta}$ is exactly the mean curvature of the geodesic sphere $S(x, r)$ at $y$. It is easy to see that if $-b^{2} \leq K \leq-a^{2}$, then $\frac{\theta^{\prime}}{\theta} \geq(n-1) a$. 
Theorem 1.6.1. (Solution of Dirichlet problem). For any $\varphi \in C^{0}(\partial \widetilde{M})$, there exists a unique harmonic function $U \in C^{\infty}(\widetilde{M}) \cap C^{0}(\bar{M})$ where $\bar{M}=\widetilde{M} \cup \partial \widetilde{M}$, such that $\left.U\right|_{\partial \widetilde{M}}=\varphi$.

Proof. (See $[\mathrm{A}][\mathrm{AS}]$ for more details.) Consider the polar coordinates system $(r, \theta)$ on $\widetilde{M}$ at the reference point $x$. Extend $\varphi$ to $\widetilde{M} \backslash\{x\}$ by $\varphi(r, \theta)=\varphi(\theta)$. Then average $\varphi$ by

$$
\widetilde{\varphi}(y)=\frac{\int_{\widetilde{M}} \chi\left(d^{2}(y, z)\right) \varphi(z) d z}{\int_{\widetilde{M}} \chi\left(d^{2}(y, z)\right) d z}
$$

where $0 \leq \chi \leq 1, \chi \in C_{0}^{\infty}(\mathbb{R})$, supp $\chi \subset[-1,1]$ and $d z$ is the Riemannian volume. One can prove that (i) $\widetilde{\varphi}(y) \rightarrow \varphi(y)$, as $y \rightarrow \infty$, (ii) $|\Delta \widetilde{\varphi}| \leq$ $C_{1} e^{-a r}$. Compare this with $(*)$. For some constants $C_{2}$ and $\delta$ small enough, one has $\Delta\left(\widetilde{\varphi}+C_{2} e^{-\delta r}\right) \leq 0 \leq \Delta\left(\widetilde{\varphi}-C_{2} e^{-\delta r}\right)$. Hence one can use $\widetilde{\varphi}+C_{2} e^{-\delta r}$ and $\widetilde{\varphi}-C_{2} e^{-\delta r}$ as barrier functions and apply the Perron method to get a harmonic function $u$ satisfying $\widetilde{\varphi}-C_{2} e^{-\delta r} \leq u \leq \widetilde{\varphi}+C_{2} e^{-\delta r}$.

1.6.2. Existence of the Green functions. The above calculation shows that if $\delta$ is small enough, then $\Delta\left(e^{-\delta r}\right) \leq 0 \leq \Delta\left(e^{-\frac{1}{\delta} r}\right)$. A result of geometry $[\mathrm{AS}]$ (actually a corollary of the maximum principle) tells us that if there exists a bounded positive superharmonic function, then there exists a global Green function $G(x, y)$, which satisfies

1. $G(x, y)=G(y, x), \quad \Delta_{y} G(x, y)=0, x \neq y$.

2. $G(x, y) \geq 0$.

3. $G(x, y) \sim \begin{cases}\log \frac{1}{r} \text { as } y \rightarrow x & , \quad \operatorname{dim} M=2, \\ r^{2-n} \text { as } y \rightarrow x & , \quad \operatorname{dim} M>2 .\end{cases}$

1.6.3. The Poisson kernel. Recall the Harnack Principle [AS]: Let $o \in \widetilde{M}$ and $v \in S_{o} \widetilde{M}$, if $u_{1}, u_{2}$ are two positive harmonic functions on the cone $c(\theta, v)=\{y \in \widetilde{M} \mid \angle(\overrightarrow{o y}, v) \leq \theta\}$, and $\left.u_{1}\right|_{\overline{c(\theta, v)} \cap \partial \widetilde{M}}=\left.u_{2}\right|_{\overline{c(\theta, v)} \cap \partial \widetilde{M}}=0$. Then there exists a constant $c>0$, such that

$$
c^{-1} \frac{u_{1}(o)}{u_{2}(o)} \leq \frac{u_{1}(x)}{u_{2}(x)} \leq c \frac{u_{1}(o)}{u_{2}(o)}
$$

As an easy corollary of the Harnack principle, for each $\xi \in \partial \widetilde{M}$ the following limit exists

$$
K(x, y, \xi)=\lim _{z \rightarrow \xi} \frac{G(y, z)}{G(x, z)}
$$


and satisfies:

1. $K(x, y, \xi)>0$,

2. $K(x, x, \xi)=1$,

3. $\left.K(x, y, \xi)\right|_{\partial M \backslash\{\xi\}}=0$.

$K(x, y, \xi)$ is called the Poisson kernel. By the Harnack principle, one can also prove that the Poisson kernels are unique.

1.6.4. Harmonic measures. For each $x \in \widetilde{M}$ and each $f \in C^{0}(\partial \widetilde{M})$ let $F(y)$ be the solution of the Dirichlet problem of $f$. Then $L_{x} f \stackrel{\text { def }}{=} F(x)$ gives a positive linear functional. It follows from the Riesz representation theorem that there exists a unique measure $\nu_{x}$ on $\partial \widetilde{M}$, such that for all $f \in C^{0}(\partial \widetilde{M}), F(x)=\int_{\partial \widetilde{M}} f(\xi) d \nu_{x}(\xi)$. By the uniqueness of the Poisson kernel, one can prove that $\frac{d \nu_{y}}{d \nu_{x}}(\xi)=K(x, y, \xi)$. The measure $\nu_{x}$ is called the harmonic measure at $x$.

1.7. Construction of invariant measures of the geodesic flow. As we noted in 1.4.3, invariant measures of $g^{t}$ on $S M$ are in a 1-1 corresondence with $\Gamma$-invariant measures on $\partial \widetilde{M} \times \partial \widetilde{M}$. First we construct, from the Patterson-Sullivan measure $\mu_{x}$ and harmonic measure $\nu_{x}, \Gamma$-invariant measures on $\partial \widetilde{M} \times \partial \widetilde{M}$.

1.7.1.. The Bowen-Margulis measure $\mu$. Define a measure $d U$ on $\partial \widetilde{M} \times$ $\partial \widetilde{M}$ by $d U(\xi, \eta)=e^{\delta \beta_{x}(\xi, \eta)} d \mu_{x}(\xi) \times d \mu_{x}(\eta)$ where $d \mu_{x}$ is the PattersonSullivan measure, $\beta_{x}(\xi, \eta)=\rho_{x, \xi}(y)+\rho_{x, \eta}(y)$ for any $y$ on the geodesic from $\xi$ to $\eta$ (one can check that this definition does not depend on the choice of $y$ ). By definition, for $\gamma \in \Gamma$,

$$
\begin{aligned}
\frac{d U(\gamma \xi, \gamma \eta)}{d\left(\gamma^{*} U\right)(\gamma \xi, \gamma \eta)} & =\frac{e^{\delta \beta_{x}(\gamma \xi, \gamma \eta)} d \mu_{x}(\gamma \xi) d \mu_{x}(\gamma \eta)}{e^{\delta \beta_{x}(\xi, \eta)} e^{\delta \rho_{\gamma^{-1}, \xi}(x)+\delta \rho_{\gamma}-1_{x, \eta}(x)} d \mu_{x}(\gamma \xi) d \mu_{x}(\gamma \eta)} \\
& =1 .
\end{aligned}
$$

Thus $d U$ is $\Gamma$-invariant on $\partial \widetilde{M} \times \partial \widetilde{M}$. Now define the Bowen-Margulis measure $\mu$ on $S \widetilde{M}$ by $d \mu(\xi, \eta, t)=d U(\xi, \eta) d t$ and then project it down to $S M$ (one can only do this because $d U$ is $\Gamma$-invariant). We denote the projected measure by the same symbol $\mu$. This is a $g^{t}$-invariant measure on $S M$, which we shall discuss in later detail.

Remark. The traditional construction of the Bowen-Margulis measure involves theory from hyperbolic dynamics. Observe that the construction here uses nothing from dynamics. One can check that, although the Patterson-Sullivan measure $\mu_{x}$ depends on the initial point $x_{0}(1.5)$, however, the measure $\mu$ does not depend on the choice of $x_{0}$ or $x$. 
1.7.2. The harmonic measure $\boldsymbol{\nu}$. The harmonic measure $\nu$ on $S M$ was first constructed by Ledrappier through Gibbs theory [L2]. The following is a more direct construction given by Kaimanovich ([Kam3]).

Let

$$
G_{x}(\xi, \eta)=\lim _{\substack{y_{1} \rightarrow \xi \\ y_{2} \rightarrow \eta}} \frac{G\left(y_{1}, y_{2}\right)}{G\left(x, y_{1}\right) G\left(x, y_{2}\right)},
$$

and define the measure $d \Lambda$ on $\partial \widetilde{M} \times \partial \widetilde{M}$ by $d \Lambda(\xi, \eta)=G_{x}(\xi, \eta) d \nu_{x}(\xi) d \nu_{x}(\eta)$ where $d \nu_{x}$ is the harmonic measure. By definition, for $\gamma \in \Gamma$,

$$
\begin{aligned}
\frac{d \Lambda}{d\left(\gamma^{*} \Lambda\right)}(\gamma \xi, \gamma \eta) & =\frac{G_{x}(\gamma \xi, \gamma \eta)}{G_{x}(\xi, \eta)} \frac{d \nu_{x}}{d \nu_{\gamma^{-1}} x}(\xi) \frac{d \nu_{x}}{d \nu_{\gamma^{-1} x}}(\eta) \\
& =\frac{G_{x}(\nu \xi, \nu \eta)}{G_{x}(\xi, \eta)} K\left(\gamma^{-1} x, x, \xi\right) K\left(\gamma^{-1} x, x, \eta\right) \\
& =1 \quad(\text { see }(1.6 .3)) .
\end{aligned}
$$

The corresponding invariant measure $d \nu(\xi, \eta, t)=d \Lambda(\xi, \eta) \cdot d t$ on $S \widetilde{M}$ and its projection on $S M$ are called the harmonic measure of the geodesic flow $g^{t}$. One can also check that this measure does not depend on the choice of $\nu_{x}$.

1.7.3.. The Liouville measure. This is the natural Liouville invariant measure corresponding to the contact structure of the geodesic flow,

$$
\int_{S M} f d m=\int_{M}\left(\int_{S_{x} M} f(v) d v\right) d x
$$

where $d x$ is the Riemannian volume on $M$ and $d v$ is the Lebesgue measure on $S_{x} M$.

If $M$ is a locally symmetric space of negative curvature, then the measures $m, \nu$ and $\mu$ all coincide. But in general they are not in the same measure class for a arbitrary manifold of negative curvature. In fact we have the following conjecture.

Conjecture (Katok [K], Sullivan [S1]). Any two of the above three measure classes coincide if and only if $M$ is locally symmetric.

\subsection{Other descriptions of the three measures.}

1.8.1. As equilibrium states. By Gibbs theory, for any Hölder function $\varphi: S M \rightarrow \mathbb{R}$, there exists a unique probability measure $\mu_{\varphi}$ called the 
equilibrium state of $\varphi$ on $S M$, which is invariant and ergodic under $g^{t}$. Moreover,

$$
h_{\mu_{\varphi}}\left(g^{t}\right)+\int \varphi d \mu_{\varphi}=\sup _{\alpha \in U(S M)}\left\{h_{\alpha}\left(g^{t}\right)+\int \varphi d \alpha\right\}
$$

where $U(S M)$ is the set of $g^{t}$-invariant probability measures on $S M$. Two Hölder functions $\varphi_{1}, \varphi_{2}$ have the same equilibrium state if and only if there exists a function $\psi$ on $S M$ such that $\varphi_{1}(v)-\varphi_{2}(v)=\left.\frac{d}{d t}\right|_{t=0} \psi\left(g^{t} v\right)$. The Bowen-Margulis measure $\mu$ is the equilibrium state of the zero function, which gives the measure of maximal entropy, $h_{\mu}=h_{\text {top }}$. The Harmonic measure $\nu$ is the equilibrium state of the function

$$
\tau(v)=\left.\frac{d}{d t}\right|_{t=0} K(v(0), v(t), v(\infty)),
$$

with entropy given by $h_{\nu}=\int_{S M} \tau(v) d \nu$. The Liouville measure $m$ is the equilibrium state of $\operatorname{tr} U(v)=\left.\frac{d}{d t}\right|_{t=0} \operatorname{det}\left(d g^{t}\left(E^{s}(v)\right)\right)$ with entropy given by $h_{m}=\int_{S M} \operatorname{tr} U d m$. This formula is known as the Pesin formula.

1.8.2. Equidistribution theorems. (see $[\mathrm{PP}])$ For any closed geodesic $\tau$, denote by $\lambda(\tau)$ the period of $\tau$. Then for any $f \in C^{0}(S M)$,

$$
\frac{\sum_{\lambda(\tau) \leq T} \int_{0}^{\lambda(\tau)} f\left(g^{t} v\right) d t}{\sum_{\lambda(\tau) \leq T} \lambda(\tau)} \longrightarrow \int_{S M} f d \mu \quad \text { as } T \rightarrow \infty .
$$

More generally, for any Hölder function $\varphi: S M \rightarrow \mathbb{R}$,

$$
\lim _{T \rightarrow \infty} \frac{\sum_{\lambda(\tau) \leq T} e^{\int_{0}^{\lambda(\tau)} \varphi(t) d t} \cdot \int_{0}^{\lambda(\tau)} f\left(g^{t} v\right) d t}{\sum_{\lambda(\tau) \leq T} \lambda(\tau) e^{f_{0}^{\lambda(\tau)} \varphi(t) d t}}=\int_{S M} f d \mu_{\varphi} .
$$

\subsection{Brownian motion along foliations and harmonic measures of} foliations ([Ga]).

Let $\mathcal{F}$ be any foliation on a compact manifold $M$ equipped with a leafwise Riemannian metric. Then we have a Laplace operator $\Delta^{L}$ on each leaf $L$. For each $x \in M$, let $P_{t}(x, y)$ be the heat kernel (see [Ga]) of $\Delta^{L}$ on $L_{x}$ (the leaf through $x$ ). Then for each $f \in C^{0}(M)$, define $T_{t} f(x) \stackrel{\text { def }}{=}$ 
$\int_{L_{x}} f(y) P_{t}(x, y) d y$, where $d y$ is the Riemannian volume on $L_{x}$. If $m$ is a measure on $M$, define $D(t) m$ by $\int_{M} f d(D(t) m)=\int_{M} D_{t} f d m$ for all $f \in C^{0}(M)$. The set of probability measures $\mathcal{E}(M)$ on $M$ is a nonempty convex set. $D(t)$ is a one-parameter family of continuous affine mappings on $\mathcal{E}(M)$. By the Markov-Kakutani fixed point theorem [Ga], there exists a measure $m$, such that $D(t) m=m$.

1.9.1. Definition. If $D(t) m=m$ for all $t$, then $m$ is called a harmonic measure for the foliation $\mathcal{F}$.

Proposition 1.9.2. ([Ga] $) m$ is harmonic if and only if

(i) $\int_{M} \Delta^{L} f d m=0$ for any bounded measurable function $f$ on $M$ which is $C^{2}$ in the leaf direction, or equivalently,

(ii) For any flow box $E$ of $\mathcal{F}, d m=K(s, y) d y d \sigma(s)$ where $K(s, y)$ is a harmonic function for each $s, d \sigma$ is a Borel measure along the transversal directions, and dy is the Riemannian volume along the leaves.

The leaf path ergodic theorem 1.9.3. ([Ga]) Let $\left\{\omega_{t}\right\}$ be the set of Brownian paths lying on the leaves of the foliation $\mathcal{F}$. Let $m$ be any harmonic ergodic measure on $M$. Then for any $f \in L^{1}(m)$,

$$
\lim _{T \rightarrow \infty} \frac{1}{T} \int_{0}^{T} f\left(\omega_{t}\right) d t=\int_{M} f d m
$$

A harmonic measure $m$ is said to be ergodic if $M$ cannot be split into two disjoint measurable leaf saturated sets with intermediate measure.

1.9.4. Unique ergodicity of the $W^{s s}$ foliation. A foliation is said to be uniquely ergodic if it has only one ergodic harmonic measure. If $M$ is a manifold of negative curvature then on $S M$ there exists a natural horospherical foliation $W^{s s}$. Its leaf passing through $v \in S M$ is defined by $W^{s s}(v)=\left\{w \in S M \mid \lim _{t \rightarrow \infty} d\left(g^{t} v, g^{t} w\right)=0\right\}$.

Theorem 1.9.4. ([L3] [Y2]) The horospherical foliation $W^{\text {ss }}$ of a negatively curved manifold $M$ is uniquely ergodic, with harmonic measure $d w^{s s}=d \mu_{x} \cdot d x$, where $d \mu_{x}$ is the Patterson-Sullivan measure on $S_{x} M$ and $d x$ is the Riemannian volume on $M$.

Proof. 1) $d \omega^{s s}$ is a harmonic measure.

(i) Define a vector field $Y$ on $M$ by

$$
Y(y)=\int_{S_{y} M} \varphi X(v) d \mu_{y}(v)=\int_{S_{x} M}\left[\varphi X e^{-h \rho_{x, v}(y)}\right] d \mu_{x}(v)
$$


where $\varphi$ is any $C^{1}$ function on $S M$ and $X$ is the geodesic spray. Then

$$
\begin{aligned}
\left.\operatorname{div}\right|_{y=x} Y & =\left.\int_{S_{x} M} \operatorname{div}\right|_{y=x}\left[\varphi X e^{-h \rho_{x, v}(y)}\right] d \mu_{x}(v) \\
& =\int_{S_{x} M}[\dot{\varphi}+(h-\operatorname{tr} U) \varphi] d \mu_{x}
\end{aligned}
$$

$(\because \operatorname{div} X=\operatorname{tr} U)$. Thus, using Green's formula

$$
\int_{S M}[\dot{\varphi}+(h-\operatorname{tr} U) \varphi] d w^{s s}=0 .
$$

(ii) Consider the function $\Phi(y)=\int_{S_{y} M} \varphi d \mu_{y}$ on $M$. Its Laplacian is given by

$$
\begin{aligned}
\left.\Delta \Phi\right|_{y=x} & =\int_{S_{y} M} \Delta\left[e^{-h \rho_{x, v}(y)} \varphi\right] d \mu_{x}(v) \\
& =\int_{S_{x} M}[\Delta \varphi+h(h-\operatorname{tr} U) \varphi+2 h \dot{\varphi}] d \mu_{x}
\end{aligned}
$$

Thus $\int_{S M}[\Delta \varphi+h(h-\operatorname{tr} U) \varphi+2 h \dot{\varphi}] d \omega^{s s}=0$. We also know that $\Delta \varphi=$ $\Delta^{s s} \varphi-\ddot{\varphi}+\dot{\varphi} \operatorname{tr} U$. If $\varphi \equiv$ const., then $\int_{S M}(h-\operatorname{tr} U) d \omega^{s s}=0$, and $h=\int_{S M} \operatorname{tr} U d \omega^{s s}$. Combining everything, one obtains $\int_{S M} \Delta^{s s} \varphi d \omega^{s s}=0$. It follows that $d \omega^{s s}$ is a harmonic measure.

2) Uniqueness. Consider the $W^{s s}$ foliation of any Anosov flow. By Sullivan and Williams ([SW]), the leaves of $W^{s s}$ have polynomial growth.

By Kaimanovich [Kam2], if all leaves of a foliation $\mathcal{F}$ have polynomial growth, then no leaf supports non-constant bounded harmonic functions. Thus all its harmonic measures $m$ must be holonomy invariant (i.e., locally, we have $d m=d y \cdot d \sigma(s)$ where the function $K(s, y)$ in proposition 1.9.2 is equal to 1). By Bowen and Marcus [BM], strong-stable foliations of Anosov flow has unique holonomy invariant measure.

1.9.5. Unique ergodicity of the $W^{s}$ foliation. Let $W^{s}$ be the weak stable foliation of the geodesic flow $g^{t}$ on a negatively curved manifold. Let $d \omega^{s}$ be the measure on $S M$, given by $\int_{S M} f d \omega^{s}=\int_{M}\left(\int_{S_{x} M} f d \nu_{x}\right) d x$ for all $f \in C^{o}(S M)$, where $d x$ is the Riemannian volume on $M$ and $d \nu_{x}$ is the harmonic measure.

Theorem 1.9.5. $d \omega^{s}$ is the unique ergodic harmonic measure of the $W^{s}$ foliation.

Proof. (1) $d \omega^{s}$ is harmonic. 
(i) By Green's formula, for all $\varphi \in C^{2}(S M)$,

$0=\int_{M} \Delta\left(\int_{S_{x} M} \varphi d \nu_{x}\right) d m(x)=\int_{M} \int_{S_{x} M}(\Delta \varphi+2\langle\nabla \varphi, \nabla \log K\rangle) d m(x)$

where $K$ is the Poisson kernel (see 1.6.3).

(ii) Again by Green's formula,

$$
\begin{aligned}
0 & =\int_{M} \operatorname{div}\left(\int_{S_{x} M} \nabla \varphi d \nu_{x}\right) d m(x) \\
& =\int_{M}\left(\int_{S_{x} M}(\Delta \varphi+\langle\nabla \varphi, \nabla \log K\rangle) d \nu_{x}\right) d m(x) .
\end{aligned}
$$

Combining (i), (ii) we have $\int_{S M} \Delta \varphi d \omega^{s}=0$ and $\int_{S M}\langle\nabla \varphi, \nabla \log K\rangle d \omega^{s}=$ 0 . Therefore $\omega^{s}$ is a harmonic measure of $W^{s}$.

(2) Uniqueness. Given any other ergodic harmonic measure $\sigma$ of the $W^{s}$ foliation and any function $f \in C^{0}(S M)$, by the leaf path ergodic theorem, for $\sigma$-a.e. leaf $W^{s}\left(x_{0}, \xi\right), \xi \in \partial \widetilde{M}$, for all $(y, \xi) \in W^{s}\left(x_{0}, \xi\right)$ and $P_{y}$ almost every path $\omega$ starting at $y$, we have (I): $\int_{S M} f d \sigma=\lim _{T \rightarrow \infty} \frac{1}{T} \int_{0}^{T} f(\widetilde{\omega}(t), \xi) d t$. Also given any other $\omega^{s}$-typical leaf $W^{s}\left(x_{0}, \eta\right)$, for $P_{y}$ almost any path $\omega$ starting at $y$, we have (II): $\int_{S M} f d \omega^{s}=\lim _{T \rightarrow \infty} \frac{1}{T} \int_{0}^{T} f(\omega(t), \eta) d t$. Consider $\omega^{s}$-typical path $\omega(t)$ and a lift of $\omega$ into the universal cover $\widetilde{\omega}$ starting at $y$ such that $\widetilde{\omega}(t) \rightarrow e \in \partial \widetilde{M}, \quad e \neq \xi, \quad e \neq \eta$; it is easy to see that $d_{\widetilde{\omega}(t)}((\widetilde{\omega}(t), \xi),(\widetilde{\omega}(t), \eta)) \longrightarrow 0(t \rightarrow \infty)$. Thus by (I), (II) $\int_{S M} f d \sigma=$ $\int_{S M} f d \omega^{s}$ for all $f \in C^{0}(S M)$. So $\sigma=\omega^{s}$.

\section{A survey of recent results.}

\subsection{Some integral formulas.}

Notations. In addition to the notations in (1.3), we fix some more notations here.

$m$ Liouville measure on $S M$

$\mu$ Bowen-Margulis measure on $S M$.

$\nu$ Harmonic measure on $S M$.

$\mu_{x}$ Patterson-Sullivan measure on $\partial \widetilde{M}$ or $S_{x} M$.

$\nu_{x}$ Harmonic measure on $\partial \widetilde{M}$ or $S_{x} M$.

$\omega^{s}$ Harmonic measure $d x d \nu_{x}$ of the $W^{s}$ foliation.

$\omega^{s s}$ Harmonic measure $d x d \mu_{x}$ of the $W^{s s}$ foliation.

$\Delta^{s}\left(\Delta^{s s}\right)$ Laplacian along the $W^{s}$ (resp. $\left.W^{s s}\right)$ foliation.

$\nabla^{s}\left(\nabla^{s s}\right)$ Gradient along the $W^{s}$ (resp. $\left.W^{s s}\right)$ foliation. 
Proposition 2.1.1. ([L3], [Kn], [Y2]) $\int_{S M}[\dot{\varphi}+(h-\operatorname{tr} U) \varphi] d \omega^{s s}=0$.

Proof. It follows from the proof of theorem 1.9.4.

Proposition 2.1.2. ([Y2]) $\int_{S M}[\dot{\varphi}+[\tau(v)-\operatorname{tr} U(v)] \varphi] d \omega^{s}=0$.

Proof. Consider $Y(x)=\int_{S_{x} M} \varphi X(v) d \nu_{x}(v)$. Then $\operatorname{div} Y(x)=\int_{S_{x} M}[\dot{\varphi}+$ $\varphi(\tau(v)-\operatorname{tr} U)] d \nu_{x}$. But by Green's formula, $\int_{M} \operatorname{div} Y(x) d x=0$.

Proposition 2.1.3. ([Y3]) $\int_{S M}\left[\Delta^{s} \varphi+\left\langle\nabla^{s} \varphi, \nabla^{s} \log g\right\rangle\right] d m=0$.

Proof. Here $g$ is the density of the conditional measure $m^{s}$ of Liouville $m$ along the $W^{s}$-foliation, characterized by

i) $\frac{g(w)}{g(v)}=\lim _{t \rightarrow \infty} \frac{J_{s}^{t}(w)}{J_{s}^{t}(v)}$, for $w \in W^{s s}(v)$

ii) $g\left(g^{t} v\right)=\frac{1}{J_{s}^{t}(v)}$ where $J_{s}^{t}(v) \triangleq \operatorname{det}\left(\left.d g^{t}\right|_{W^{s s}(v)}\right)$.

Consider the vector field $\nabla^{s} \varphi$ on $S M$ and its flow $\Phi_{t}$. For any small open $W^{s}$-flow box $E$ we have $\left.\frac{d}{d t}\right|_{t=0} \operatorname{vol}\left(\Phi_{t} E\right)=\int_{E}\left(\Delta^{s} \varphi+\left\langle\nabla^{s} \varphi, \nabla^{s} \log g\right\rangle\right) d m$. Since this is true for all flow boxes $E$, the formula is also true for $S M$. Yet $\left.\frac{d}{d t}\right|_{t=0} \operatorname{vol}\left(\Phi_{t}(S M)\right)=0$.

\subsection{Some corollaries.}

Proposition 2.2.1 ([L3], [Y2]). If $\omega^{s}$ or $\omega^{s s}$ coincide with $\mu, m$, or $\nu$, then $M$ is asymptotically harmonic (i.e. $\operatorname{tr} U \equiv h$ ).

Proof. If $\omega^{s s}$ coincides with a $g^{t}$-invariant measure, then (2.1.1) gives

$$
\int_{S M}(h-\operatorname{tr} U) \varphi d \omega^{s s} \equiv 0
$$

for all $\varphi \in C^{1}(S M)$. Thus $h \equiv \operatorname{tr} U$. Similarly, if $\omega^{s s}$ coincides with a $g^{t}$ invariant measure, then (2.1.2) gives $\operatorname{tr} U(v) \equiv \tau(v)$ for all $v \in S M$. Then by theorem A and lemma 3.2 of $[\mathrm{Y} 1], \operatorname{tr} U \equiv h$.

Proposition 2.2.2. If for all $x \in \widetilde{M}, \mu_{x}=\nu_{x}, \mu_{x}=m_{x}$, or $\nu_{x}=m_{x}$, then $M$ is asymptotically harmonic.

Proof. 1) If $\mu_{x}=m_{x}$ or $\nu_{x}=m_{x}$ for all $x \in \widetilde{M}$, then, respectively $\omega^{s s}=m$ or $\omega^{s}=m$. So the result follows from proposition 2.2.1.

2) If $\mu_{x}=\nu_{x}$ for all $x \in \widetilde{M}$, then $\omega^{s s}=\omega^{s}$. By the formulas (2.1.1) and (2.1.2), $\int_{S M}[h-\tau(v)] \varphi d \omega^{s} \equiv 0$ for all $\varphi \in C^{1}(S M)$. It follows that $h \equiv \tau(v)$. It follows that $M$ is asymptotically harmonic: in fact, $\mu_{x}=\nu_{x}$ for all $x \in \widetilde{M}$ implies that $e^{-h \rho_{x, \xi}(y)}=K(x, y, \xi)$ (see (1.5), (1.6)). Thus $0=\Delta K(x, y, \xi)=\Delta e^{-h \rho_{x, \xi}(y)}=h(h-\operatorname{tr} U) e^{-h \rho_{x, \xi}(y)}$. 
2.3. Margulis function. Margulis $[\mathrm{M}]$ proved the existence of the following limit:

$$
c(x)=\lim _{R \rightarrow \infty} \frac{\operatorname{vol}(S(x, R))}{e^{h R}},
$$

where $S(x, R)$ is a geodesic sphere in $\widetilde{M}$ and $h$ is the topological entropy of the geodesic flow.

Proposition 2.3.0 ([Y3]). $\frac{c(y)}{c(x)}=\int_{S_{x} M} e^{-h \rho_{x, \xi}(y)} d \mu_{x}(\xi)$. In particular $c(y)$ is a smooth function.

Proof. Look at the family of measures $\mu_{x}^{R}$ on $\partial \widetilde{M}$ given by $d \mu_{x}^{R} \triangleq P_{x}^{*}$. $\left(\frac{\left.d y\right|_{S_{x}(R)}}{e^{h R}}\right)$, i.e. the push forward by $P_{x}$ of the normalized Riemannian volume on $S(x, R)$ where $P_{x}: S(x, R) \rightarrow \partial \widetilde{M}$ is defined by $y \mapsto$ the positive asymptotic of the geodesic from $x$ to $y$. Let $\bar{\mu}_{x}=\lim _{R_{k} \rightarrow \infty} \mu_{x}^{R_{k}}$ be a weak limit and let $\bar{\mu}_{y} \triangleq \lim _{R_{k} \rightarrow \infty} \mu_{y}^{R_{k}}$. Then it is easy to see that $\frac{d \bar{\mu}_{y}}{d \bar{\mu}_{x}}=e^{-h \rho_{x, \xi}(y)}$. By the uniqueness of Patterson-Sullivan measure (see 1.9.4), the above limit is unique and $\bar{\mu}_{x}=c \cdot \mu_{x}$ for a constant $c$. Thus $c(y)$, which is the total mass of $\bar{\mu}_{y}$, must satisfy $\frac{c(y)}{c(x)}=\int_{S_{x} M} e^{-h \rho_{x, \xi}(y)} d \mu_{x}(\xi)$. The smoothness of $c(y)$ follows from the smoothness of the Busemann function.

Proposition 2.3.1 ([Y3]). If $c(x) \equiv$ const., and $\operatorname{dim} M=2$, then $M$ has constant negative curvature.

Proof. By formula 2.1.1 if $\varphi \equiv 1$, we get $h=\int_{S M} \operatorname{tr} U d \omega^{s s}$ and if $\varphi \equiv \operatorname{tr} U$, we get $h^{2}=\int_{S M}\left[-\operatorname{tr} \dot{U}+(\operatorname{tr} U)^{2}\right] d w^{s s}$. If $\operatorname{dim} M=2$, then by the Riccatti equation, $-\operatorname{tr} \dot{U}+\operatorname{tr} U^{2}+K=0$, one has

$$
h^{2}=\frac{\int_{M}-K(x) c(x) d x}{\int_{M} c(x) d x} .
$$

If $c(x) \equiv$ const., then $h^{2}=\frac{{ }^{{ }_{M}-K(x) d x}}{\operatorname{vol}(M)}=-2 \pi E / \operatorname{vol}(M)$ where $E$ is the Euler characteristic number of $M$. (Gauss-Bonnet). By $[\mathrm{K} 1], h^{2}=$ $-2 \pi E / v(M)$ if and only if $K \equiv$ const.

Remark. In higher dimension, if $c(x) \equiv$ const., then for all $x \in \widetilde{M}, h \equiv$ $\int_{S_{x} M} \operatorname{tr} U(v) d \mu_{x}$.

Proposition 2.3.2 ([Y3], [Kn]). If $M$ is a negatively curved closed manifold, then

$$
h^{2}=\int_{S M}\left[R^{H}(v)-R(\pi(v))+\operatorname{Ric}(v)\right] d \omega^{s s}
$$


where $R^{H}(v)$ is the scalar curvature of the horosphere $H(v)$ at $v, R(\pi(v))$ is the scalar curvature of $M$ at $\pi(v)$ and $\operatorname{Ric}(v)$ is the Ricci curvature of $M$ at $v$. If $\operatorname{dim} M=3$, then $h^{2}=\int_{S M}[\operatorname{Ric}(v)-R(\pi(v))] d \omega^{s s}$.

Proof. Let $K^{H}$ be the sectional curvature of $H(v)$ with respect to the induced Riemannian metric. Then, for any orthonormal vectors $X, Y$ in $T_{\pi(v)} H(v)$, by the Gauss equation,

$$
K^{H}(X, Y)=K(X, Y)+\langle U(v) X, X\rangle\langle U(v) Y, Y\rangle-\langle U(v) X, Y\rangle\langle U(v) Y, X\rangle
$$

where $U(v)$ is the second fundamental form. From this, one gets $R^{H}(v)=$ $R(\pi(v))+(\operatorname{tr} U)^{2}-\operatorname{tr} U^{2}-2 R i c(v)$. Combining this with the Ricatti equation, $-\dot{U}+U^{2}+\operatorname{Ric}=0$ one gets $R^{H}(v)=R(\pi(v))+(\operatorname{tr} U)^{2}-\operatorname{tr} \dot{U}-\operatorname{Ric}(v)$. Now using the identity $h^{2}=\int_{S M}\left[-\operatorname{tr} \dot{U}+(\operatorname{tr} U)^{2}\right] d \omega^{s s}$ we get the first statement. If $\operatorname{dim} M=3$, by Conne's leaf-wise version of Gauss-Bonnet theorem [Co], $\int_{S M} R^{H}(v) d \omega^{s s}=0$. Therefore $h^{2}=\int_{S M}\left(\operatorname{Ric}(v)-R(\pi(v)) d \omega^{s s}\right.$.

2.4. Flip-invariance of the Patterson-Sullivan and harmonic measures $\mu_{x}, \nu_{x}$. Associated to the fibration $\left\{S_{x} M\right\}_{x \in M}$ of $S M$, there exists a canonical system of conditional measures $\left\{\bar{\mu}_{x}\right\}_{x \in M}$ and $\left\{\bar{\nu}_{x}\right\}_{x \in M}$ for the Bowen-Margulis measure $\mu$ and harmonic measure $\nu$.

Question. (Kifer and Ledrappier [KL]). Is $\bar{\mu}_{x}$ (resp. $\bar{\nu}_{x}$ ) equivalent to the Patterson-Sullivan measure $\mu_{x}$ (resp. harmonic measure $\left.\nu_{x}\right)$ ?

Proposition 2.4.1 ([Y5]). (1) $\bar{\mu}_{x}=\mu_{x} / c(x)$ for a.e. $x \in M$ (with respect to the projected measure of $\mu$ onto $M$ ) if and only if $M$ is asymptotically harmonic. (2) The same result is true for $\bar{\nu}_{x}$. (3) If $\bar{\mu}_{x}\left(\right.$ resp. $\left.\bar{\nu}_{x}\right)$ is in the same measure class as $\mu_{x}$ (resp. $\nu_{x}$ ) for a.e. $x \in M$, then $\mu=m$ (resp. $\nu=m)$.

The proof follows from the following lemmas.

Lemma 2.4.2. If $m$ is a Borel probability measure on $S M$ which is flip invariant (i.e.invariant under the flip map $f: S M \rightarrow S M, v \mapsto-v$ ), then for $m$-a.e. $x \in M$, the conditional measure $\bar{m}_{x}$ on $S_{x} M$ is also flip invariant.

Proof. This follows from the flip invariance of the partition $\left\{S_{x} M\right\}_{x \in \bar{M}}$ and the uniqueness of conditional measures.

Lemma 2.4.3. Both the Bowen-Margulis measure $\mu$ and the harmonic measure $\nu$ are flip invariant.

Proof. This follows from the construction in section 1.7. 
Lemma 2.4.4. If for all $x \in M, \mu_{x}$ (resp. $\left.\nu_{x}\right)$ is flip-invariant, then $\mu=m($ resp. $\nu=m)$.

Proof. We need to prove that $\frac{d m_{x}}{d \mu_{x}}(\xi)$ is finite for all $\xi \in \partial \widetilde{M}$. Assume the contrary, then there is at least one $\xi \in \partial \widetilde{M}$, such that

$$
\varlimsup_{\epsilon \rightarrow 0} \frac{m_{x}\left(D_{x}(\xi, \epsilon)\right)}{\mu_{x}\left(D_{x}(\xi, \epsilon)\right)}=0
$$

For any other point $\eta \in \partial \widetilde{M}$ and any point $y$ on the geodesic $\overrightarrow{\xi \eta}$, since $\mu_{x}$ and $\mu_{y}$ (resp. $m_{x}$ and $m_{y}$ ) are equivalent with positive Radon-Nikodym derivatives, one has

$$
\varlimsup_{\epsilon \rightarrow 0} \frac{m_{y}\left(D_{y}(\xi, \epsilon)\right)}{\mu_{y}\left(D_{y}(\xi, \epsilon)\right)}=0 \text { by }(*) .
$$

Since $m_{y}$ and $\mu_{y}$ are flip invariant, (**) gives $\varlimsup_{\epsilon \rightarrow 0} \frac{m_{y}\left(D_{y}(\eta, \epsilon)\right)}{\mu_{y}\left(D_{y}(\eta, \epsilon)\right)}=0$ for any $\eta \in \partial \widetilde{M}$. Similarly if $\frac{\lim }{\epsilon \rightarrow 0} \frac{\mu_{x}\left(D_{x}(\xi, \epsilon)\right)}{m_{x}\left(D_{x}(\xi, \epsilon)\right)}=\infty$ for some $\xi \in \partial \widetilde{M}$ then $\underline{\lim _{\epsilon \rightarrow 0}} \frac{\mu_{x}\left(D_{x}(\eta, \epsilon)\right)}{m_{x}\left(D_{x}(\eta, \epsilon)\right)}=\infty$ for all $\eta \in \partial \widetilde{M}$.

Since both $\mu_{x}$ and $m_{x}$ are finite their Radon-Nykodym derivative must be finite somewhere and hence everywhere. Thus $\mu_{x}$ is equivalent to $v_{x}$ for all $x$ and $\mu=m$.

Lemma 2.4.5 ([Y5]). If $\mu_{x}$ is flip invariant, then the Margulis function $c(x) \equiv$ const.

Proof. By (2.1.1), $\int_{S M}[\dot{\varphi}+(h-\operatorname{tr} U) \varphi] d \omega^{s s}=0$ for all $\varphi \in C^{2}(S M)$. Let $\varphi$ be any $C^{2}$ function on $M$ and then lift it to a function on $S M$ which we denote by the same notation, since $\mu_{x}$ is flip-invariant, $\int_{S M} \dot{\varphi} d \omega^{s s}=$ $\int_{M}\left(\int_{S_{x} M} \dot{\varphi} d \mu_{x}\right) d x=0$. Thus $\int_{S M}(h-\operatorname{tr} U) \varphi d \omega^{s s}=0$ for all $\varphi \in C^{2}(M)$. It follows easily that $\int_{S_{x} M}(h-\operatorname{tr} U) d \mu_{x}=0$ for all $x \in M$. But $\Delta c(x)=$ $h \int_{S_{x} M}(h-\operatorname{tr} U) d \mu_{x}$, so $\Delta c(x) \equiv 0, c(x) \equiv$ constant.

Proof of Proposition 2.4.1. If $\mu_{x}=\bar{\mu}_{x}$ for a.e. $x \in M$, then $\mu_{x}$ is flip invariant by (2.4.2) and (2.4.3). By Lemma 2.4.4, $\mu=m$. Therefore $\bar{\mu}_{x}=m_{x}$. By Lemma 2.4.5. $c(x) \equiv$ const. Thus $\mu_{x} \equiv m_{x}$. Therefore $\omega^{s s}=\mu=m$, and $M$ is asymptotically harmonic (see 2.2.1).

\subsection{Entropy rigidity in dimension 2.}

In $\operatorname{dim} M=2$ we have the following special result. For any metric $g$, there exists a positive function $\rho$ on $M$ such that $g=\rho g_{0}$ where $g_{0}$ is a 
metric of constant curvature. The relationship between various operators under this conformal transformation is the following

(1) Curvature: $K_{g}=\frac{1}{\rho}\left[K_{0}+\frac{1}{2} \Delta^{g_{0}}(\log \rho)\right]$.

(2) Volume element: $d m_{g}=\rho d m_{g_{0}}$.

(3) Laplacian: $\Delta^{g}=\frac{1}{\rho} \Delta^{g_{0}}$.

In particular, $g$ and $g_{0}$ have the same harmonic functions.

Proposition 2.5.1. ([L2]). If $\nu=\mu$, then $K^{g} \equiv$ const.

Proof. If $\nu=\mu$, by the variational principle (see for example $[\mathrm{PP}]$ ), there exists a function $f$ on $S M$ such that $h_{g}-\tau(v)=\dot{f}(v)$ (see (1.8.1) for the definition of $\tau(v))$, here $h_{g}$ is the topological entropy. It follows that $e^{-h_{g} \rho_{x, \xi}(y)+f(y, \xi)-f(x, \xi)}=K(x, y, \xi)$. Therefore

$$
\begin{gathered}
\Delta^{g} f+\left\|\nabla^{g} f\right\|^{2}+h_{g}\left(h_{g}-\operatorname{tr} U\right)+2 h_{g} \dot{f}=0 . \\
\frac{1}{\rho}=\|\nabla \log K\|^{2}=h_{g}^{2}+2 h_{g} \dot{f}+\|\nabla f\|^{2} .
\end{gathered}
$$

Combining these we get

$$
\Delta^{g} f-h \operatorname{tr} U=-\frac{1}{\rho}
$$

Integrating both sides of (2) with respect to the Liouville measure $m_{g}$ of $g$, one gets

$$
\begin{aligned}
& \int \frac{1}{\rho(y)} d m^{g}(y)=h_{g}^{2}+\int_{S M}\|\nabla f\|^{2} d m_{g} \geq h_{g}^{2} . \quad \text { On the other hand, } \\
& \int \frac{1}{\rho(y)} d m^{g}(y)=\frac{\int_{M} \frac{1}{\rho(y)} \rho(y) d x}{\int_{M} \rho(y) d y}=\frac{v_{0}(M)}{\int_{M} \rho(y) d y}=\frac{v_{0}(M)}{v^{g}(M)}
\end{aligned}
$$

But by [K1], this is only possible when $\rho$ is constant.

Other important invariants of a metric $g$ are the Kamanovich entropy $\beta_{g}$, and the speed of Brownian motion $\alpha_{g}$,

$$
\beta_{g} \stackrel{\text { def }}{=} \int_{S M}\left\|\nabla^{g} \log K^{g}\right\|^{2} d \omega_{g}^{s}, \quad \alpha_{g} \stackrel{\text { def }}{=} \int_{S M} \tau^{g}(v) d \omega_{g}^{s} .
$$

By [Kam1], [L1], [L2], $4 \lambda_{g}^{1} \leq \beta_{g}=h_{\nu}^{g} \cdot \alpha_{g} \leq h_{g}^{2}$, where $h_{\nu}^{g}$ is the metric entropy of the harmonic measure $\nu$. 
Proposition 2.5.2. For any metric $g=\rho g_{0}$ of negative curvature, if $g$ and $g_{0}$ have the same volume, then $h_{g} \geq h_{0}$ and $h_{\nu}^{g} \geq h_{0}$ with equality iff $\rho \equiv 1$. (Here $h_{0}$ is the topological entropy of $g_{0}$ and $h_{g}$ is the topological entropy of $g, h_{g}^{\nu}$ is the metric entropy of the geodesic flow of $g$ with respect to its harmonic measure $\nu$ ).

Proof. Observe that 1) $\left\|\nabla^{g} \log K\right\|^{2}=\frac{1}{\rho}$, and 2) $\tau^{g}(v)=\left.\frac{d}{d t}\right|_{t=0} \log K(v(0)$, $v(t), v(\infty)=\frac{1}{\sqrt{\rho}}$. It follows that

$$
\begin{aligned}
& \beta^{g}=\int_{S M}\left\|\nabla^{g} \log K\right\|^{2} d \omega_{g}^{s}=\int_{S M} \frac{1}{\rho} d \omega_{g}^{s}=\frac{\operatorname{vol}^{g_{0}}(M)}{\operatorname{vol}^{g}(M)} \text { and } \\
& \alpha^{g}=\int_{S M} \tau^{g}(v) d \omega_{g}^{s}=\int_{S M} \frac{1}{\sqrt{\rho}} d \omega_{g}^{s}=\frac{\int_{M} \sqrt{\rho} d^{g_{0}} x}{\int_{M} \rho d^{g_{0}} x} .
\end{aligned}
$$

As $\operatorname{vol}^{g_{0}}(M)=\operatorname{vol}^{g}(M)=1$, we obtain $\beta^{g}=\beta^{g_{0}}=h_{0}^{2}, \alpha^{g} \leq \alpha^{g_{0}}=h_{0}$. But $\beta_{g}=h_{\nu}^{g} \cdot \alpha_{g} \leq\left(h^{g}\right)^{2}$. Therefore $h^{g} \geq h_{0}, h_{\nu}^{g} \geq h_{0}$, with equality if and only if $\rho \equiv$ const.

Re-interpretation of $\alpha^{g}$. . The quantity $\alpha^{g}=\frac{{ }^{\int_{M} \sqrt{\rho} d^{g_{0}} x}}{\mathrm{vol}^{g}(M)}$ was first introduced by Katok [K1] as a measurement of the deviation from constant curvature. It turns out that it is the asymptotic speed of Brownian motions on $\widetilde{M}: \alpha^{g}=\lim _{t \rightarrow \infty} \frac{1}{t} d(\omega(t), x)$, for almost all Brownian motions $\omega(t)$.

Proposition 2.5.3 ([K1]). Let $g_{1}$ be a metric of negative curvature on a closed manifold $M, \nu$ be a Borel probability invariant measure of the geodesic flow of $g_{1}$. Then for any other metric $g_{2}, h^{g_{2}} \geq \frac{h_{\nu}^{g_{1}}}{\sigma\left(g_{2}\right)}$ where $\sigma\left(g_{2}\right) \triangleq \int_{S^{g_{1} M}}\|v\|_{g_{2}} d \nu$. In particular, if $g_{2}=\rho g_{1}$ for some positive function $\rho$, then $h^{g_{2}} \geq \frac{h_{\nu}^{g_{1}}}{\int_{S^{g_{1}} M} \sqrt{\rho} d \nu}$.

Corollary 2.5.4 ([K1]). For any metric $g=\rho g_{0}$ on a closed surface $M$ where $g_{0}$ is a metric of constant negative curvature, if $\mathrm{vol}^{g}(M)=$ $\operatorname{vol}^{g_{0}}(M)=1$, then $h_{m}^{g} \leq h_{0}$, with equality iff $\rho \equiv 1$.

Corollary 2.5.5 ([K1]). If $h^{g}=h_{m}^{g}$ (i.e., the Bowen-Margulis measure coincides with the Liouville measure), then $K_{g} \equiv$ const.

Proof. By corollary (2.5.4) and proposition (2.5.2), $h^{g}=h_{m}^{g}$ implies that $h^{g}=h_{m}^{g}=h_{0}$.

By the same argument, one can prove the following

Corollary 2.5.6. If $h_{m}^{g}=h_{\nu}^{g}$ (i.e., the Liouville measure coincides with the Lebesgue measure), then $K_{g} \equiv$ const. 


\subsection{Entropy-like invariants and bottom of the Laplace spec-} trum. The purpose of this section is to collect all entropy like invariants together and show their relationships, providing the simplest possible proofs.

2.6.1. Bottom of spectrum $\lambda_{1}$. Let $\lambda_{1}$ be the bottom of the spectrum of the Laplace operator $-\Delta$ on $L^{2}(\widetilde{M})$. By the Rayleigh's principle, $\lambda_{1}=$ $\inf _{f \in C_{0}^{1}} \frac{\int_{M}|\nabla f|^{2}}{\int_{M} f^{2}}$, where $C_{0}^{1}$ is the set of all $C^{1}$ functions on $\widetilde{M}$ with compact support.

Proposition 2.6.1 ([L1]). $\lambda_{1} \leq \frac{h^{2}}{4}$

Proof. For any $s>\frac{h}{2}, f(y) \stackrel{\text { def }}{=} e^{-s d(x, y)} \in L^{2}(\widetilde{M})$. Therefore $\lambda_{1} \leq$ $\frac{\int_{M} \| \nabla f \mid}{\int_{M} f^{2}}=s^{2}$.

2.6.2. Kaimanovich entropy $\beta$. ([Kam1]) It is defined by

$$
\beta \triangleq \lim _{t \rightarrow \infty}-\frac{1}{t} \ln P(t, x, \widetilde{\omega}(t))
$$

for almost all Brownian motion (see also section 2.5).

Proposition 2.6.2 ([L2]). $\lambda_{1} \leq \frac{\beta}{4}$ with equality if and only if $M$ is asymptotically harmonic.

Proof. (For more detail, see [Kam1] and [L2]).

$$
\begin{aligned}
\beta & =\lim _{t \rightarrow \infty}\left(-\frac{1}{t} \int_{\widetilde{M}} P(t, x, y) \log P(t, x, y) d y\right) \quad([\operatorname{Kam} 1]) \\
& =\lim _{t \rightarrow \infty}-\frac{1}{t} \int_{0}^{t}\left\{\int_{\bar{M}}[1+\log P(s, x, y)] \frac{\partial}{\partial s} P(s, x, y) d y\right\} d s \\
& =\lim _{t \rightarrow \infty}-\frac{1}{t} \int_{0}^{t}\left[\int_{\bar{M}}[1+\log P(s, x, y)] \Delta_{y} P(s, x, y) d y\right] d s \\
& =\lim _{t \rightarrow \infty} \frac{1}{t} \int_{0}^{t}\left(\int_{\widetilde{M}} \frac{\|\nabla P(s, x, y)\|^{2}}{P} d y\right) d s \\
& =\lim _{t \rightarrow \infty} 4 \cdot \frac{1}{t} \int_{0}^{t}\left(\int_{\widetilde{M}}\|\nabla \sqrt{P(s, x, y)}\|^{2} d y\right) d s \\
& \geq 4 \lambda_{1} \quad\left(\because \quad \int_{\bar{M}} P(t, x, y) d y=1\right) .
\end{aligned}
$$

In [L4] it is proved that $\beta=4 \lambda_{1}$ if and only if $M$ is asymptotically harmonic. 
2.6.3. Asymptotic speed of Brownian motion $\alpha$. $\alpha$ is defined by $\alpha \triangleq$ $\lim _{t \rightarrow \infty} \frac{1}{t} d(\omega(t), x)$ for almost all Brownian path $\omega(t)$ starting from $x \in \widetilde{M}$ $([\operatorname{Kam} 1])$.

Proposition 2.6.3 ([L1]). $\beta=h_{\nu} \alpha$.

Corollary 2.6.4. $\alpha \leq h_{\nu}$.

Proof. By Kaimanovich [Kam1],

$$
\beta=\int_{S m}\|\nabla \log K\|^{2} d \omega^{s} \quad \text { and } \quad \alpha=\int_{S M} \tau(\nu) d \omega^{s}
$$

Thus $\beta \geq \alpha^{2}$.

2.7. A problem of Green. Let $M$ be a closed manifold of negative sectional curvature. Then the mean curvature of horospheres $\operatorname{tr} U$ is a welldefined function on $S M$. In [G], L. Green proved that if $\operatorname{tr} U(v) \equiv u(x)$ for some positive function $u(x)$ and for all $v \in S_{x} M$, i.e. the mean curvature of the horospheres depends only on the base point and $\operatorname{dim} M=2$, then $M$ has constant curvature. One can actually prove the following generalization.

Theorem 2.7 ([Y4]). Let $M$ be a closed manifold of negative curvature such that $\operatorname{tr} U(v) \equiv u(x)$, for some positive function $u(x)$ on $M$ and for all $v \in S_{x} M$. Then

(1) $M$ is asymptotically harmonic.

(2) The geodesic flow of $M$ is $C^{\infty}$ conjugate to the geodesic flow of a locally symmetric space.

Proof. (1) The first observation is that the Margulis function $c(x)$ satisfies a certain Laplace equation. By definition

$$
c(y)=\int_{S_{x} m} e^{-h \rho_{x, \xi}(y)} d \mu_{x}(\xi)
$$

and so

$$
\begin{aligned}
\Delta c(y) & =h \int_{S_{x} M}(h-\operatorname{tr} U) e^{-h \rho_{x, \xi}(y)} d \mu_{x}(\xi) \\
& =h(h-u(y)) c(y) .
\end{aligned}
$$

Therefore $\|\nabla \log c(y)\|^{2}+\Delta(\log c(y))=h(h-u(y))$. Thus, by Green's formula and Pesin's formula $([\mathrm{P}]), \int_{M}\|\nabla \log c(y)\|^{2} d y=h\left(h-h_{m}\right) \operatorname{vol}(M)$. 
(2) The argument of [FL] can be generalized to show that if $\operatorname{tr} U(v)$ is smooth on $S M$, then the geodesic flow of $M$ is smoothly conjugate to the geodesic flow of a locally symmetric space. Now if $\operatorname{tr} U(v) \equiv u(x)$, then by (2.7.1), $u(x)$ is smooth on $M$; thus $\operatorname{tr} U(v)$ is smooth on $S M$. By smooth conjugacy, $h=h_{m}$, and so $\|\nabla \log c(x)\| \equiv 0$, and $c(x)$ is a constant. From (2.7.1) again, $h \equiv u(x)$.

\subsection{Some conjectures and open problems.}

The first "Geometric Rigidity Conference" in 1984 at MSRI resulted in among other things two problem lists $[\mathrm{BK}],[\mathrm{Hu}]$. The first one was centered on manifolds of nonpositive curvature and the other one was about the rigidity of group actions and cocycles. Since the appearance of $[\mathrm{BK}]$, some problems on manifolds of nonpositive curvature were solved and some new problems were also posed. I take this opportunity to cite some open problems around manifolds of strictly negative curvature. Most of the problems are well known among experts and some of the problems might also have come across other persons' minds. No originality is claimed by the author. Throughout this section, $M$ denotes a closed manifold of negative sectional curvature.

Conjecture 2.8.1. Prove that if any two of the three measures $\mu$ (BowenMargulis), $\nu$ (harmonic), $m$ (Liouville) coincide, then $M$ is asymptotically harmonic. This conjecture is true in the 2-dimensional case. See section 2.5 and the references there.

Conjecture 2.8.2. Let $M, N$ be two closed manifolds of negative curvature. Prove that if there exists a continuous time-preserving conjugacy $\varphi: S M \rightarrow S N$ of the geodesic flows, then $\varphi$ is $C^{1}$.

One would also hope that $M$ and $N$ were isometric. Again this is true if $\operatorname{dim} M=2([\mathrm{C}][\mathrm{O}])$. The higher dimensional cases are open even under the additional assumption that $\varphi$ is $C^{\infty}$ and $N$ is locally symmetric. In this case, if $M$ is asymptotically harmonic and if $\operatorname{dim} M=3$ or 4 , then we know that $M$ is a locally symmetric space (Ledrappier, Hamenstädt, unpublished).

Conjecture 2.8.3. If the Margulis function is constant, then $M$ is asymptotically harmonic. (This is true if $\operatorname{dim} M=2$ (see proposition 2.3.1).)

Conjecture 2.8.4. If the projection of the Bowen-Margulis measure $\mu$ on $S M$ to $M$ coincides with the Riemannian volume then $\mu=m$.

For background material, see $[\mathrm{KKW}]$. This problem is closely related to the study of critical points of the topological entropy. The conjecture is true if $\operatorname{dim} M=2$. 
Problem 2.8.5. Study the variational property of various entropy-like invariants.

In dimension 2, one has a clear picture, namely, entropy-like invariants in $\S 2.6$ attain a maximum or a minimum value at a metric of constant curvature. The higher dimensional picture seems to be much more complicated. See $[\mathrm{BCG}]$ for related results.

Problem 2.8.6. From the Laplacian spectrum, one can read the volume and total scalar curvature of $M$, and also the length spectrum. If $M$ is locally symmetric, then by the trace formula, the knowledge of Laplacian spectrum is equivalent to the knowledge of length spectrum. This is no longer true for a general manifold of negative curvature. Is it possible to read the volume and total scalar curvature from the marked length spectrum? This is probably easy to show for asymptotically harmonic manifolds. If this were true, then it would imply that an asymptotically harmonic manifold homotopic to a manifold of constant negative curvature must also have constant curvature.

Problem 2.8.7. By [M], [Y2], one knows that the volume of a geodesic ball $B(x, R)$ satisfies

$$
\operatorname{vol}(B(x, R))=c(x) \frac{e^{h R}}{h}+\epsilon(x, R)
$$

where $c(x)$ is in general non constant. What is the asymptotic behavior of the error term $\epsilon(x, R)$ ? Is it true that in general, $\lim _{R \rightarrow \infty} \frac{\log |\epsilon(x, R)|}{R}<h$ ? This is actually a problem of the correlation coefficients for the mixing of the geodesic flow. If $M$ is locally symmetric, the answer is yes ([Mo]).

Problem 2.8.8. Is there any connection between the (local) Einstein condition of a Einstein manifold $M$ and the (global) dynamical invariants of the geodesic flow?

Problem 2.8.9. If $M$ is a closed manifold of negative curvature and $N$ is a closed manifold of constant negative curvature. If $f: M \rightarrow N$ is a $C^{2}$ diffeomorphism which maps the geodesics in $M$ into curves in $N$ which are reparameterizations of geodesics in $N$, then the author can prove that there must exist a $C^{2}$ time-preserving conjugacy between the geodesic flows of $M$ and $N$. In particular, this implies that if $\operatorname{dim} M=2$, then $M$ and $N$ must be isometric. Is this true if both $M$ and $N$ have variable negative curvature?

\section{Acknowledgement}

Anatoly Katok first suggested to me the idea of expanding my seminar talks into a survey. I thank him for his patience in reading through my 
original manuscript and offering important corrections. I also thank Luis Barreira for pointing out a number of errors in the original version.

\section{References}

[A] M. T. Anderson, The Dirichlet problem at infinity for manifolds of negative curvature, J. Diff. Geom. 18 (1983), 701-721.

[AS] M. T. Anderson, R. Schoen, Positive harmonic functions on complete manifolds of negative curvature, Ann. Math. 121 (1985), 429-446.

[Ba] W. Ballman, Nonpositively curved manifolds of higher rank, Ann. of Math. (2) 122 (1985), 597-609.

[BS] K. Burns, R. Spatzier, Manifolds of nonpositive curvature and their buildings, IHES Publ. 65 (1987), 35-59.

[BBE] W. Ballmann, M. Brin, P. Eberlein, Structure of manifolds of nonpositive curvature, I, Ann. of Math. 122 (1985), 171-203.

[BBS] W. Ballmann, M. Brin, R. Spatzier, Structure of manifolds of nonpositive curvature, II, Ann. of Math. 122 (1985), 205-235.

[BCG] G. Besson, G. Courtois, S. Gallot, Sur les minima localux de l'entropie topologique, preprint.

[BF] Y. Benoist, F. Labourie, Sur les difféomorphisms d'Anosov affines à feuilletages stable et instable différentiables, Inv. Math. 111 (1993), 285-308.

[BFL] Y. Benoist, F. Foulon, F. Labourie, Flots d'Anosov á distribution stable et instable différentiables, J. Am. Math. Soc. 5 (1992), 33-74.

[BK] K. Burns, A. Katok, Manifolds with non-positive curvature, Erg. Th. \& Dyn. Syst. 5 (1985), 307-317.

[BM] R. Bowen, B. Marcus, Unique ergodicity of horospherical foliations, Isr. J. Math. 62 (1977), 43-69.

[C] C. Croke, Rigidity for surfaces of nonpositive curvature, Comment. Math. Helv. 65 (1990), 150-169.

[CE] J. Cheeger, D. Ebin, Comparison theorems in Riemannian geometry, NorthHolland Publ. Company, 1975.

[Co] A. Connes, A survey of foliations and operator algebras, AMS Proc. Symp. Pure Math., v. 38, 521-628.

[E] P. Eberlein, Lattices in manifolds of nonpositive curvature, Ann. of Math. (2) 111 (1980), 435-476.

[F] R. Feres, Geodesic flows on manifolds of negative curvature with smooth horospheric foliations, Erg. Th. \& Dyn. Syst. 11 (1991), 653-686.

[FL] P. Foulon, F. Labourie, Sur les variétés asymptotiquement harmoniques, Inv. Math. 109 (1992), 97-111.

[FK1] R. Feres, A. Katok, Invariant tensor fields of dynamical systems with pinched Lyapunov exponents and rigidity of geodesic flows, Erg. Th. \& Dyn. Syst. 9 (1989), 427-432.

[FK2] R. Feres, A. Katok, Anosov flows with smooth foliation and rigidity of geodesic flows in three dimensional manifolds of negative curvature, Erg. Th. \& Dyn. Syst. 10 (1990), 657-670.

[G] M. Gromov, Large Riemannian manifolds, LNM 1201 (1986), 108-122.

[Ga] L. Garnett, Foliations, the ergodic theorem and Brownian motion, J. Fun. Anal. 51 (1983), 285-311. 
[GT] M. Gromov, W. Thurston, Pinching constants for hyperbolic manifolds, Inv. Math. 89 (1987), 1-12.

[Ha1] B. Hasselblatt, Horospheric foliations and relative pinching, Preprint.

[Ha2] B. Hasselblatt, Bootstrapping regularity of the Anosov splitting, AMS Proc. 115 (1992), 817-819.

[HK] S. Hurder, A. Katok, Differentiability, rigidity and Godbillor-Vey classes for Anosov flows, IHES Publ. 72 (1990), 5-61.

[HP] M. Hirsch, C. Pugh, Smoothness of horocycle foliations, J. Diff. Geom. 10 (1975), 225-238.

[Hu] S. Hurder, Problems on rigidity of group actions and cocycles, Erg. Th. \& Dyn. Syst. 5 (1985), 473-484.

[H1] U. Hamenstädt, An explicit description of the harmonic measure, Math. Z. 205 (1990), 287-299.

[H2] U. Hamenstädt, Time-preserving conjugacy of geodesic flows, Erg. Th. \& Dyn. 12 (1992), 67-74.

[H3] U. Hamenstädt, Metric and topological entropy of geodesic flows, preprint.

[H4] U. Hamenstädt, Entropy rigidity of compact locally symmetric spaces, Ann. of Math. (2) 131 (1990), 35-51.

[H5] U. Hamenstädt, A geometric characterization of compact locally symmetric spaces, J. Diff. Geom. 34 (1991), 455-464.

[K] M. Kanai, Geodesic flows on negatively curved manifolds with smooth stable and unstable foliations, Erg. Th. \& Dyn. Syst. 8 (1988), 215-239.

[K1] A. Katok, Entropy and closed geodesics, Erg. Th. \& Dyn. Syst. 2 (1982), 339367.

[K2] A. Katok, Four applications of conformal equivalence to geometric and dynamics, Erg. Th. \& Dyn. Syst. 8 (1988), 139-152.

$[\mathrm{Kl}] \mathrm{W}$. Klingenber, Riemannian geometry, de Gruyter Studies in Mathematics, 1982.

[Kam1] V. A. Kaimanovich, Brownian motion and harmonic functions on covering manifolds, an entropy approach, Soviet Math. Doklady 33 (1985), 812-816.

[Kam2] V. A. Kaimanovich, Brownian motion on foliations: entropy, invariant measures, mixing, Funct. Anal. Appl. 22 (1988), 326-328.

[Kam3] V. A. Kaimanovich, Invariant measures of the geodesic flow and measures at infinity on negatively curved manifolds, Ann. I. H. P. 53 (1990), 361-393.

[KKW] A. Katok, G. Knieper, H. Weiss, Formulas for derivative and critical points of topological entropy for Anosov and geodesic flows, Commun. Math. Phys. 138 (1991), 19-31.

[Kn] G. Knieper, Horospherical measure and rigidity of manifolds, preprint.

[KL] Y. Kifer, F. Ledrappier, Hausdorff dimension of harmonic measures on negatively cruved manifolds, T. A. M. S. 318 (1990), 685-704.

[L1] F. Ledrappier, Ergodic property of Brownian motion on covers of compact negatively curved manifolds, Bol. Soc. Bras. Mat. 19 (1988), 115-140.

[L2] F. Ledrappier, Harmonic measures and Bowen-Margulis measures, Isr. J. Math. 71 (1990), 275-287.

[L3] F. Ledrappier, Ergodic properties of the stable foliations, preprint.

[L4] F. Ledrappier, A heat kernel characterization of asymptotic harmonicity, preprint.

[M] G. A. Margulis, Applications of ergodic theory to the investigation of manifolds of negative curvature, Func. Anal. Appl. 3 (1969), 335-336. 
[Mo] C. C. Moore, Exponential decay of correlation coefficients for geodesic flows, Group representation, ergodic theory, operator algebras, and mathematical physics, MSRI publication, Springer-Verlag, 1987.

[O] J. Otal, Le spectre marqué des surfaces à courbure negative, Ann. of Math. (2) 131 (1990), 151-162.

[P] Y. Pesin, Equation for the entropy of a geodesic flow on a compact Riemannian manifold without conjugate points, Math. Notes 24 (1978), 796-805.

[PP] W. Parry, M. Pollicott, Zeta functions and the periodic orbit structure of hyperbolic dynamics, Astérisque (1990) 187-188.

[S1] D. Sullivan, The Dirichlet problem at infinity for a negatively curved manifold, J. Diff. Geom. 18 (1983), 723-732.

[S2] D. Sullivan, The density at infinity of a discrete group of hyperbolic motions, Publ. Math. IHES. 50 (1979), 225-250.

[SW] D. Sullivan, R. Williams, On the homology of attractors, Topology 15 (1976), 259-262.

[Y1] C. B. Yue, On Sullivan's conjecture, Random \& Comp. Dyn. 1 (1992), 131-145.

[Y2] C. B. Yue, Brownian motion on Anosov foliations and manifolds of negative curvature, to appear in J. Diff. Geom.

[Y3] C. B. Yue, Integral formulas for Laplacian along the unstable foliation, Erg. Th. \& Dyn. 11 (1991), 803-819.

[Y4] C. B. Yue, On Green's conjecture, preprint.

[Y5] C. B. Yue, Conditional measure and flip invariance of Bowen-Margulis and harmonic measure on manifolds of negative curvature, to appear in Erg. Th. \& Dyn. Syst.

Department of Mathematics, Penn State Univerity, Univerity Park, Pa 16802

E-mail address: yue@math.psu.edu 